\title{
Effects of pressure and noise on the stability of photoacoustic signals of trace gas components
}

\author{
Zhizhen Zhu ${ }^{1,2}$, Jing Luo ${ }^{3}$, Jiaxiang Liu' ${ }^{1}$, Yonghua Fang ${ }^{1 *}$ \\ (1. Key Laboratory of Environmental Optics and Technology, Anhui Institute of Optics and Fine Mechanics, \\ Chinese Academy of Sciences, Hefei 230031, China; \\ 2. University of Science and Technology of China, Hefei 230026, China; \\ 3. Huaibei Normal University, Huaibei 235000, Anhui, China)
}

\begin{abstract}
In essence, photoacoustic spectroscopy (PAS) technology is based on the thermal effect of gas infrared absorption and the acoustic theory of photoacoustic (PA) cell. PAS technology has a good application effect on environmental monitoring in agriculture. In this study, carbon monoxide and sulfur dioxide were used as examples to explain the potential application of PAS technology and analyze the influence mechanism of pressure and noise on the PA signal. The relationship between PA signal amplitude and the concentration of gas was determined by calibration. The pressure and noise characteristics were experimentally studied, and the relationship between the PA signal and pressure \& noise was obtained. The theoretical analysis and experimental results not only provided a basis for further correction of the influence of pressure noise and other factors on PA signal but also provided technical support for improving the field application of trace gas non-resonance PA detection device for environmental monitoring in agriculture.
\end{abstract}

Keywords: photoacoustic spectroscopy, environmental monitoring in agriculture, trace gas, pressure, noise DOI: $10.25165 /$ j.ijabe.20201305.5749

Citation: Zhu Z Z, Luo J, Liu J X, Fang Y H. Effects of pressure and noise on the stability of photoacoustic signals of trace gas components. Int J Agric \& Biol Eng, 2020; 13(5): 187-193.

\section{Introduction}

Trace gases in livestock and poultry houses are harmful to animal health and one of the sources of air pollutants. However, it is difficult to detect trace gases in livestock and poultry houses in real-time. Although electrochemical sensors can measure hydrogen sulfide and ammonia, there is still a lack of effective means for the detection of sulfur oxide and carbon monoxide.

Gas photoacoustic spectroscopy (PAS) is a kind of trace gas detection technology based on the photoacoustic (PA) effect. As a new method of spectral detection, PAS has the advantages of high sensitivity, good stability, no consumption of carrier gas, small volume, easy to carry and on-line monitoring. At present, it has become a hot spot in the field of trace gas detection and has a good application prospect ${ }^{[1-7]}$.

In recent years, a lot of research on PAS has been carried out in some units at home and abroad. Since 2000, Kelman Company of the UK has successfully developed a Transfix on-line dissolved gas analyzer for transformer oil by using no-resonance PAS technology, which can detect 8 kinds of fault gases and micro-water content in transformer oil.

In 2011, the State Key Laboratory of Transmission and Distribution Equipment and System Safety and New Technologies of Chongqing University successfully developed an $\mathrm{SF}_{6}$

Received date: $2020-02-21 \quad$ Accepted date: 2020-08-14

Biographies: Zhizhen Zhu, PhD candidate, research interest: infrared spectral processing, Email: 734961000@qq.com; Jing Luo, PhD, research interest: photoacoustic spectroscopy, Email: 1jmfs0926@163.com; Jiaxiang Liu, PhD, research interest: infrared remote sensing, Email: luckuis@aiofm.ac.cn.

*Corresponding author: Yonghua Fang, $\mathrm{PhD}$, Professor, research interest: opto-electronic information. Key Laboratory of Environmental Optics and Technology, Anhui Institute of Optics and Fine Mechanics, Chinese Academy of Sciences, Hefei 230031, China. Tel: +86-551-65595027, Email: yhfang@ aiofm.ac.cn decomposition component detection device based on resonance PAS technology and the detection sensitivity of $\mathrm{SO}_{2}$ gas reached $7.5 \mu \mathrm{L} / \mathrm{L}^{[8]}$.

Zhang et al. ${ }^{[9]}$ analyzed the effects of PAS on the detection of trace gases, and determined the central wavelength of the band-pass filter required for $\mathrm{CO}$, $\mathrm{NO}$ and $\mathrm{H}_{2} \mathrm{~S}$ detection, thus realizing the improvement of gas absorption efficiency and cross-interference resistance.

Gong et al. ${ }^{[10]}$ analyzed the interference mechanism of water vapor in the flue gas on the measurement of nitrogen oxide, designed the test system of resonance PA detection of nitrogen oxide in flue gas, quantitatively analyzed the PA signal response generated by the fluctuation of excitation light source, and comprehensively analyzed the gas absorption rate and anti-cross-sensitivity factors.

$\mathrm{Du}$ et al. ${ }^{[1]}$ realized the rapid determination of the $\mathrm{NH}_{3}$ concentration in the top space of the soil by using the mid-infrared PAS and multiple regression analysis, which was used to study the volatilization process of soil $\mathrm{NH}_{3}$ and its influencing factors and to provides a new method for the study of the soil nitrogen cycle.

As far as the current research situation is concerned, the PAS technology has the potential for environmental monitoring in agriculture, which has basically met the requirements in the detection sensitivity and detection range. However, in practice, when the PAS technology is used in the gas monitoring of livestock and poultry houses, the measurement accuracy of PA spectra is seriously affected. It is found that the PAS method will be affected by many environmental factors in practical applications, such as temperature, humidity, pressure and vibration ${ }^{[12.13]}$. The PA detection device for environmental monitoring in agriculture will inevitably be affected by the above factors.

Carbon monoxide has been used as an example to analyze the influence mechanism of temperatures and derives the temperature correction formula using the non-resonant PA detection device 
designed by our team ${ }^{[14]}$. The correction method has provided an effective way of studying temperature correction in PA detection.

With the PA cell is closed, the changes of pressure in the PA cell will directly cause the changes in the PA signal ${ }^{[15-19]}$. In the actual experimental process, besides paying attention to the PA signal produced by the gas to be measured, various types of noise cannot be ignored ${ }^{[18]}$. Among them, the sound or vibration signal with the same frequency as the modulation frequency contained in related noise and environmental noise should be analyzed emphatically. Therefore, it is necessary to analyze the influence mechanism of pressure and noise on PA signal through theoretical analysis and experimental study, which provides technical support for the field application of non-resonant PA detection devices for environmental monitoring in agriculture.

In this study, carbon monoxide and sulfur dioxide were used as examples to explain the potential application of PAS technology and analyze the influence mechanism of pressure and noise on the PA signal.

\section{Principle of PAS}

The gas molecules to be measured in a closed container generate sound signal after being exposed to modulate light or pulse light periodically is called the gas PA effect.

The generation and detection of the PA signal are shown in Figure 1.

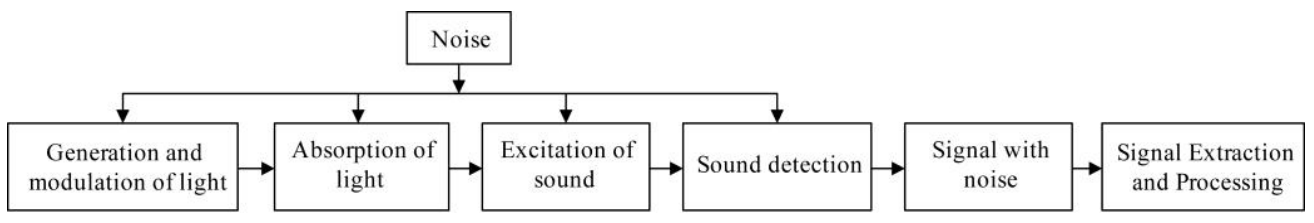

Figure 1 Schematic of the PA detection principle

After absorbing the incident light of a specific wavelength, some gas molecules in the PA cell transition from the ground state to the excited state. The molecules in the excited state collide with the molecules in the ground state, resulting in a non-radiative transition back to the ground state. The light energy absorbed by the gas changes into the translational kinetic energy between the colliding molecules, and the gas temperature increases.

According to the law of gas absorption, the increase of gas temperature in the closed space leads to an increase in gas pressure. When the incident light is modulated at a certain frequency (tens of hertz to thousands of Hertz), the air pressure is modulated at the same frequency to form a sound wave. This sound wave can be detected by a microphone installed on the PA cell and converted into an electrical signal. The generation and detection of PA signal is an energy conversion process of light, heat, sound and electricity. After the PA signal is obtained, the gas concentration can be determined by using the quantitative relationship between the gas concentration and the PA signal.

The theoretical analysis shows that the amplitude of sound pressure corresponding to the non-resonant PA detection device can be expressed by Equation (1):

$$
A_{0}(\omega)=\frac{i \alpha(\gamma-1 \oint I \vec{r}(\omega, d V)}{\omega V_{c}\left(1+\frac{i}{\omega \tau_{0}}\right)}
$$

where, $\tau_{0}$ is the thermal damping time, $\mathrm{s}$, which depends on the thermal conductivity of the gas to the wall of the PA cell. For a PA cell with radius $R$, the relationship between the thermal damping time $\tau_{0}$ and the gas density in the cell $\rho_{0}$, heat capacity at constant pressure $C p$ and thermal conductivity $\mathrm{K}$ is as $\tau_{0} \approx \rho_{0} C_{p} R^{2} / 2.5 \mathrm{~K}$.

After amplification and transformation of the microphone, the voltage amplitude of the PA signal can be expressed by Equation (2):

$$
U=S_{\min } \cdot P_{s} \cdot C_{\text {cell }} \cdot N \cdot \sigma
$$

where, $S_{\text {min }}$ is the sensitivity of the microphone, $\mathrm{mV} / \mathrm{Pa} ; P_{s}$ is the source power, W; $C_{\text {cell }}$ is the PA cell constant, $\mathrm{Pa} \cdot \mathrm{cm} / \mathrm{W}$, which generally relates to the size of PA cell and the physical constants of carrier gas, etc. $N$ is the total number of molecules per unit volume, molec $/ \mathrm{cm}^{3} ; \sigma$ is the absorption cross-section of gas, $\mathrm{cm}^{2} / \mathrm{molec}$; the product of which is the absorption coefficient $\alpha$, $\mathrm{cm}^{-1}$.
It can be seen from Equation (2) that the PA signal of gas has a linear relationship with the gas concentration when other parameters remain unchanged. Therefore, the concentration of the gas to be measured can be calculated by calibrating the equation between the PA signal and the concentration.

\section{Structure chart of PAS device}

The non-resonant PA detection device developed by our team is shown in Figure 2, which is small in size, easy to carry, and can realize on-line monitoring.

The device consists of IR source, chopper, filters, PA cell, microphone, control module, data acquisition and data processing module. The chopper realizes the intensity modulation of the infrared light source by rotating at a fixed frequency and steady speed. A narrow-band infrared light source can be obtained to excite a specific gas PA signal by using a specific narrow band filter. By controlling the rotation of the filter wheel with a stepping motor, the switching between one gas filter and another gas filter can be realized. After the gas in the PA cell is irradiated by the modulated narrow-band infrared light, the sound pressure signal is generated, and the high-sensitivity microphone is used to convert the generated sound pressure signal into an electrical signal. The data acquisition module collects the PA signal and sends the collected PA signal to the data processing module for processing. Then the gas type and concentration are analyzed. In order to analyze the influence factors of the PA signal, pressure sensors are installed on the PA detection device.

The details of PAS device are as Table 1.
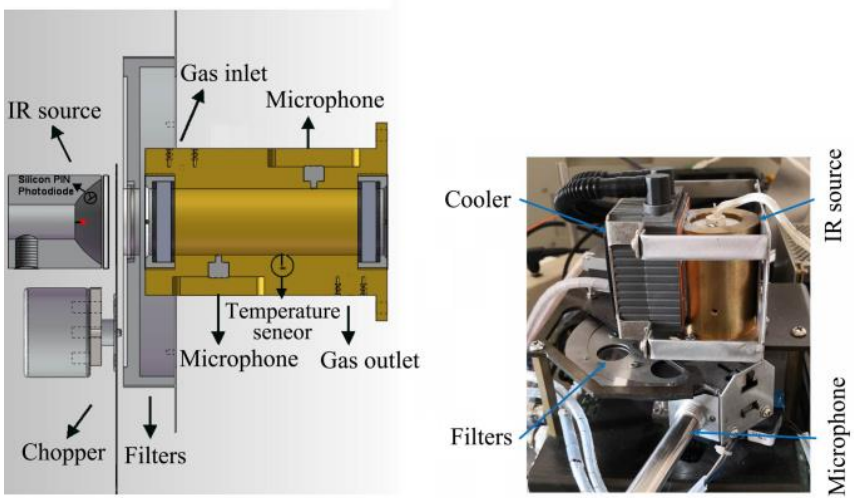

Figure 2 Photograph of the non-resonant PA detection device 
Table 1 Details of PAS device

\begin{tabular}{lc}
\hline \multicolumn{1}{c}{ Device elements } & Specific parameters \\
\hline IR source and intensity & Incoherent IR source, $19.5 \mathrm{~W}$ \\
Frequence of chopper & $30 \mathrm{~Hz}$ \\
Filter wavelength & $4.7 \mu \mathrm{m}$ for $\mathrm{CO}, 7.37 \mu \mathrm{m}$ for $\mathrm{SO}_{2}$ \\
Sensitivity of microphone & $50 \mathrm{~mW} / \mathrm{Pa}$ \\
Temperature sensor & $-40^{\circ} \mathrm{C}-100^{\circ} \mathrm{C}$
\end{tabular}

\section{Results}

\subsection{Calibration experiments for $\mathrm{CO}$ and $\mathrm{SO}_{2}$}

It can be seen from Equation (2) that, when the values of other parameters are constant, there is a linear relationship between the PA signal amplitude and concentration of the gas. By preparing several groups of standard gases with known concentrations to be measured, the corresponding PA signals are measured, and the calibration equation between the PA signal amplitude and concentration is obtained by multiple linear regression.

The calibration experiment is carried out under normal temperature and pressure. The CO standard gas of different concentrations is equipped with a mass flow controller (the carrier gas is $\mathrm{SF}_{6}$ ) and is successively charged into the PA cell for one minute. By measuring the PA signals of different concentrations of CO standard gas, the linear relationship between the PA signals and the concentration is shown in Equation (3) and Figure 3:

$$
u_{1}=0.00181 \times c_{1}+2.51719
$$

where, $u_{1}$ is the PA signal voltage, $\mathrm{V}$ and $c_{1}$ is the concentration of $\mathrm{CO}, \mu \mathrm{L} / \mathrm{L}$

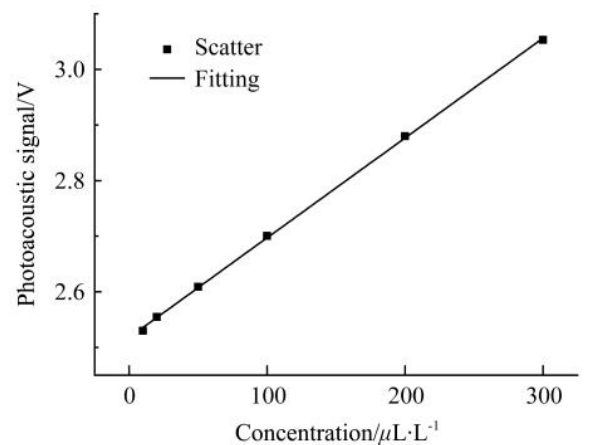

Figure 3 Schematic of relationship between concentration and photoacoustic signal of $\mathrm{CO}$

The relationship between $\mathrm{SO}_{2}$ photoacoustic signal and concentration can be obtained under the same experimental conditions and steps, as shown in Equation (4) and Figure 4.

$$
u_{2}=0.00023 \times c_{2}+1.63222
$$

where, $u_{2}$ is the PA signal voltage, $\mathrm{V}$ and $c_{2}$ is the concentration of $\mathrm{SO}_{2}, \mu \mathrm{L} / \mathrm{L}$

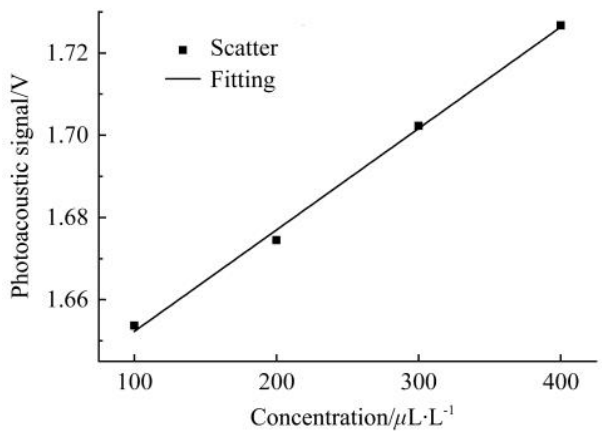

Figure 4 Schematic of relationship between concentration and photoacoustic signal of $\mathrm{SO}_{2}$

\subsection{Stability analysis of PA signal}

To meet the needs of the on-line detection of trace gases, the PA detection device needs to have good stability.

In order to verify the stability of the developed PA detection device, three groups of high, medium and low concentration gas mixtures containing $\mathrm{CO}$ and $\mathrm{SO}_{2}$ components are measured respectively for 9 consecutive hours. The concentration composition of each component in these three groups of gas mixtures is shown in Table 2. The results of continuous measurement of three groups of mixed gases by PA detection device are shown in Table 3, Table 4 and Table 5.

Table 2 Concentration composition of two mixed gases

\begin{tabular}{ccc}
\hline & \multicolumn{2}{c}{ Concentration $/ \mu \mathrm{L} \cdot \mathrm{L}^{-1}$} \\
\cline { 2 - 3 } No. & $\mathrm{CO}$ & $\mathrm{SO}_{2}$ \\
\hline 1 & 132 & 145 \\
2 & 63 & 80 \\
3 & 27 & 37 \\
\hline
\end{tabular}

Table 3 Continuous measurement results of $9 \mathrm{~h}$ of high concentration mixture

\begin{tabular}{ccccc}
\hline $\begin{array}{c}\text { Measurement } \\
\text { time/h }\end{array}$ & $\begin{array}{c}\mathrm{CO} \\
/ \mu \mathrm{L} \cdot \mathrm{L}^{-1}\end{array}$ & $\begin{array}{c}\text { Relative error } \\
/ \%\end{array}$ & $\begin{array}{c}\mathrm{SO}_{2} \\
/ \mu \mathrm{L} \cdot \mathrm{L}^{-1}\end{array}$ & $\begin{array}{c}\text { Relative error } \\
/ \%\end{array}$ \\
\hline 1 & 132.6 & 0.5 & 145.6 & 0.4 \\
2 & 134.1 & 1.6 & 160.6 & 10.8 \\
3 & 130.1 & 1.5 & 156.4 & 7.8 \\
4 & 139.0 & 5.3 & 141.8 & 2.2 \\
5 & 139.2 & 5.4 & 144.1 & 0.6 \\
6 & 133.5 & 1.1 & 151.4 & 4.4 \\
7 & 125.7 & 4.7 & 148.4 & 2.4 \\
8 & 130.2 & 1.4 & 150.5 & 3.8 \\
9 & 140.7 & 6.6 & 132.5 & 8.6 \\
\hline
\end{tabular}

Table 4 Continuous measurement results of $9 \mathrm{~h}$ of medium concentration mixture

\begin{tabular}{ccccc}
\hline $\begin{array}{c}\text { Measurement } \\
\text { time/h }\end{array}$ & $\begin{array}{c}\mathrm{CO} \\
/ \mu \mathrm{L} \cdot \mathrm{L}^{-1}\end{array}$ & $\begin{array}{c}\text { Relative error } \\
/ \%\end{array}$ & $\begin{array}{c}\mathrm{SO}_{2} \\
/ \mu \mathrm{L}^{-1} \mathrm{~L}^{-1}\end{array}$ & $\begin{array}{c}\text { Relative error } \\
/ \%\end{array}$ \\
\hline 1 & 65.8 & 4.5 & 96.3 & 20.4 \\
2 & 59.4 & 5.7 & 82.3 & 2.9 \\
3 & 64.2 & 1.9 & 95.7 & 19.0 \\
4 & 56.2 & 10.8 & 83.2 & 4.1 \\
5 & 55.1 & 12.6 & 75.8 & 5.2 \\
6 & 53.2 & 15.6 & 67.5 & 15.7 \\
7 & 56.0 & 11.1 & 59.5 & 25.7 \\
8 & 58.1 & 7.8 & 65.5 & 18.1 \\
9 & 64.3 & 2.1 & 60.0 & 25.0 \\
\hline
\end{tabular}

Table 5 Continuous measurement results of $9 \mathrm{~h}$ of low concentration mixture

\begin{tabular}{ccccc}
\hline $\begin{array}{c}\text { Measurement } \\
\text { time/h }\end{array}$ & $\begin{array}{c}\mathrm{CO} \\
/ \mu \mathrm{L} \cdot \mathrm{L}^{-1}\end{array}$ & $\begin{array}{c}\text { Relative error } \\
/ \%\end{array}$ & $\begin{array}{c}\mathrm{SO}_{2} \\
/ \mu \mathrm{L} \cdot \mathrm{L}^{-1}\end{array}$ & $\begin{array}{c}\text { Relative error } \\
/ \%\end{array}$ \\
\hline 1 & 26.0 & 3.71 & 48.6 & 31.3 \\
2 & 34.6 & 28.23 & 42.3 & 14.3 \\
3 & 32.5 & 20.38 & 40.3 & 8.9 \\
4 & 31.6 & 17.02 & 50.2 & 35.6 \\
5 & 30.2 & 11.73 & 48.4 & 30.8 \\
6 & 28.7 & 6.46 & 35.0 & 5.3 \\
7 & 27.1 & 0.15 & 27.4 & 26.0 \\
8 & 28.4 & 5.30 & 30.2 & 18.3 \\
9 & 24.0 & 11.46 & 28.9 & 22.0 \\
\hline
\end{tabular}


The relative errors of measured values corresponding to actual values are initially used to describe the stability of the system.

From Table 3, it can be seen that the concentration of two components of high concentration gas mixture is relatively stable with time, and the relative error for 9 consecutive hours is within 10.8\%; from Table 4 and Table 5, it can be seen that for low and medium concentration gas mixture, the relative error of two components concentration with time is relatively large.

Next step, the stability of PA signal is further checked.

When comparing the stability of two or more groups of data, if the unit of measurement and the average are the same, the standard deviation can be directly used for comparison. If both the unit and the average are different, instead, the ratio (relative value) of the standard deviation to the average should be used to compare.

The ratio of the standard deviation to the average is called the coefficient of variance, which is a relative index to check the stability and dispersion degree of data. Its function is mainly to reflect the degree of data dispersion on the unit average and eliminate the influence of the average on the comparison of the degree of variation of different groups of data. The coefficient of variance (CV) is expressed as Equation (5):

$$
\mathrm{CV}=\sigma / \mu
$$

where, $\sigma$ is the standard deviation and $\mu$ is the average.

The values of $\mathrm{CV}$ for $\mathrm{CO}$ and $\mathrm{SO}_{2}$ in Table 3, Table 4 and Table 5 are shown in Table 6 .

Table 6 Comparison of $\mathrm{CV}$ with different concentration

\begin{tabular}{ccc}
\hline & $\mathrm{CO}$ & $\mathrm{SO}_{2}$ \\
\hline $\mathrm{CV}$ in Table 3 & 0.034994 & 0.052698 \\
$\mathrm{CV}$ in Table 4 & 0.073016 & 0.175095 \\
$\mathrm{CV}$ in Table 5 & 0.107800 & 0.217476 \\
\hline
\end{tabular}

In principle, the smaller the value of $\mathrm{CV}$ is, the better the stability of the PA detection device.

In order to improve the performance of the PA detection device, it is necessary to deeply analyze the factors that affect the PA signal, find out the key factors that affect the sensitivity and stability of the PA signal detection, and further improve the algorithm.

\section{Influence of pressure and noise}

\subsection{Influence of Pressure on PA Signals}

The PA cell is closed, the changes of pressure in the PA cell will directly cause the changes in PA signal ${ }^{[15,19]}$. Pressure affects PA signal mainly by changing the absorption cross-section of gas molecules.

In the PA detection of trace gas, the central wavelength of $\mathrm{CO}$ filter of target gas is $4.7 \mu \mathrm{m}$, so when analyzing the influence of pressure on $\mathrm{CO}$ gas absorption cross-section, the band of $4.45 \mu \mathrm{m}$ to $4.95 \mu \mathrm{m}$ containing this central wavelength is selected for analysis.

The absorption cross-sections of a single $\mathrm{CO}$ molecule at different pressures of $0.1 \mathrm{MPa}, 0.4 \mathrm{MPa}, 0.8 \mathrm{MPa}$ and $2.8 \mathrm{MPa}$ (a temperature of $296 \mathrm{~K}$ ) are calculated using the line-by-line method combining the HITRAN2004 database, as shown in Figure 5.

It can be seen from Figure 5 that when the pressure is increased, the line width of the absorption line will be increased so that the separated absorption lines will be combined and the cross-response between different gases will be aggravated.

The dependence of total molecular absorption cross-section $\sigma_{\Sigma}$ and total absorption coefficient $\alpha_{\Sigma}=N \sigma_{\Sigma}$ on pressure for $\mathrm{CO}$ gas in the range of 4.45-4.95 $\mu \mathrm{m}$ wavelength is also calculated, as shown in Figure 6.

It can be seen from Figure 6 and Equation (2) that when the PA signal is measured, increasing the pressure is beneficial to increase the PA signal.

As can be seen from Figure 6, the relationship between total absorption coefficient $\alpha_{\Sigma}$ and pressure of CO gas in the range of 4.45-4.95 $\mu \mathrm{m}$ can be divided into three stages: 1) When the gas pressure varies from 0 to $10 \mathrm{MPa}$, the total absorption coefficient of the gas molecule shows a sharp upward trend and approximately obeys linear growth; 2) When the gas pressure varies from $10 \mathrm{MPa}$ to $30 \mathrm{MPa}$, the total absorption coefficient of gas molecules increases slowly; 3) When the pressure is greater than $30 \mathrm{MPa}$, the total absorption coefficient of the molecule is stable and basically remains unchanged. The reason for this phenomenon is that the broadening mechanism of the absorption spectra of gas molecules is different in different pressure ranges.
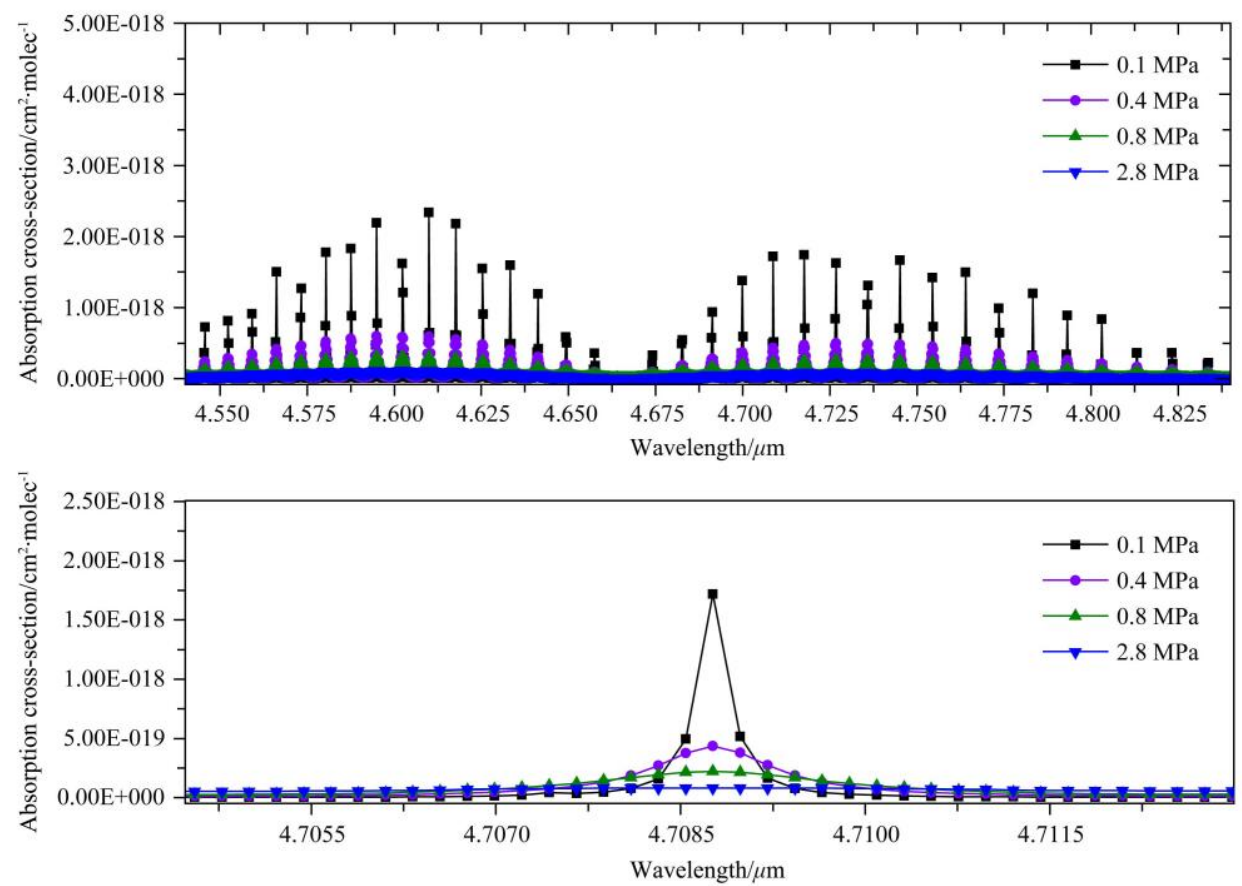

Figure 5 Schematic of absorption cross-sections of a single CO molecule at different pressures (4.45-4.95 $\mu \mathrm{m})$ 


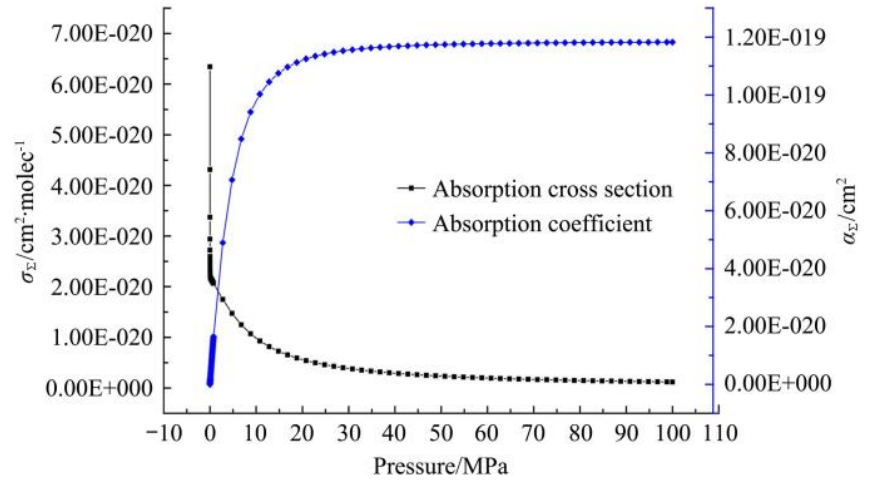

Figure 6 Variation of total absorption coefficient and total absorption cross-sections of $\mathrm{CO}(4.45-4.95 \mu \mathrm{m})$ with pressures

The influence of pressure on the PA signal is studied experimentally with $100 \mu \mathrm{L} / \mathrm{L}$ CO gas. In the experiment, the PA detection device is put into a constant temperature and humidity chamber, and the temperature is approximately $25^{\circ} \mathrm{C}$. A $100 \mu \mathrm{L} / \mathrm{L}$ mixture of carbon monoxide ( $\mathrm{SF}_{6}$ as carrier gas) is filled into the PA cell and the air inflation process lasted for two minutes and the inlet and outlet valves are closed in turn after charging. The air intake device is equipped with a pressure sensor (measuring accuracy is $100 \mathrm{~Pa}$ ). In PA measurement, the pressure of the gas intake device from the gas tank is about $0.7 \mathrm{MPa}$. According to the requirements, the gas intake device needs four return journeys, so that the pressure entering the PA detection device is $0.1 \mathrm{MPa}$ (the fluctuation is not more than $1 \%$ ). The pressure of the gas intake device fluctuates up and down each time, so the pressure of the gas entering the PA cell also fluctuates. The integration time is set to $1 \mathrm{~s}$, and the PA signal and pressure signal corresponding to the $\mathrm{CO}$ filter are collected at the same time. In order to improve the measurement precision, the gross errors of measurement results are removed using Grubbs' criterion and the testing values of repeated measurements are averaged. The PA signals of $100 \mu \mathrm{L} / \mathrm{L}$ $\mathrm{CO}$ gas corresponding to different pressures are obtained, as shown in Figure 7.

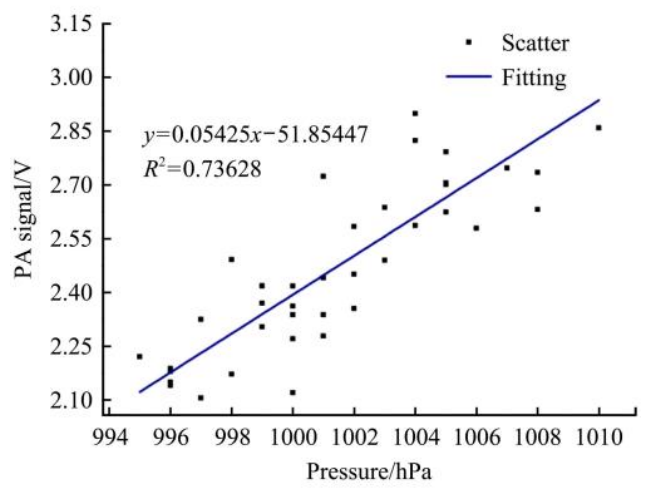

Figure 7 Schematic of variation of PA signal with pressures

As can be seen from Figure 7, the PA signal shows an upward trend with the increase of pressure, but at the same time, it can be seen that under the same pressure, there are several corresponding PA signal values. There may be two reasons for this phenomenon. One is that the pressure sensor in the intake device measures the pressure of the intake port, which does not fully represent the change of the pressure in the PA cell; the other is the influence of other factors besides the pressure. Although the PA detection device is placed in the constant temperature and humidity box, its temperature is not absolutely constant, and the constant temperature and humidity box will produce greater vibration when working and the vibration noise may also have an impact on the PA signal.

\subsection{Influence of noise on PA signals}

In the actual experimental process, besides paying attention to the PA signal produced by the gas to be measured, various types of noise cannot be ignored ${ }^{[20]}$. Noise sources in gas PA detection devices mainly include environmental noise, chopper noise, electrical noise generated by the electronic detection system, thermal fluctuation noise generated by gas Brown motion and background noise generated by window and pool wall absorbing modulated light energy, etc. ${ }^{[9,20]}$ The background signal caused by chopper noise and window pool wall absorbing modulated light energy belongs to correlated noise, while environmental noise, thermal fluctuation noise of gas Brown motion and electrical noise belong to uncorrelated noise. Uncorrelated noise can be generally eliminated by phase-locked amplification technology, however, the correlated noise and environmental noise contain the same frequency sound signal or vibration as the modulation frequency cannot be eliminated by the same method.

In addition to affecting the detection sensitivity of the system, the sound or vibration signal with the same frequency as the modulation frequency contained in the correlated noise and environmental noise will also affect the stability of the PA signal. Next, the influence of this part of the noise on the stability of the PA signal is analyzed in depth. Among them, chopper noise, environmental noise, gas Brown motion noise and electrical noise are independent of the power of the light source, while the background noise caused by the absorption of the pool wall and the window is related to the power of the light source ${ }^{[21,22]}$. When the power of the light source is constant, the background noise absorbed by the pool wall and window will not change with time. Here we focus on the analysis of source-independent noise.

Relevant experiments are carried out. High purity $\mathrm{N}_{2}$ is filled into the PA cell and the light source is turned off. At this time, the total of chopper noise, environmental noise, gas flow noise and electrical noise are measured. After the chopper is turned off, the total of environmental noise, gas flow noise and electrical noise is measured. The experimental results are shown in Figure 8. When the chopper is turned on, the mean value of equivalent PA signal caused by chopper noise, environmental noise, gas flow noise and electrical noise is $0.01398 \mathrm{~V}$, and the standard deviation is $8.1681 \times 10^{-4} \mathrm{~V}$. After turning off the chopper, the mean value of the equivalent PA signal becomes $0.01376 \mathrm{~V}$ and the standard deviation is $6.1961 \times 10^{-4} \mathrm{~V}$. It can be seen that the noise of the chopper only accounts for a small part of the source-independent noise, while the environmental noise, gas flow noise and electrical noise are the main parts of the source-independent noise.

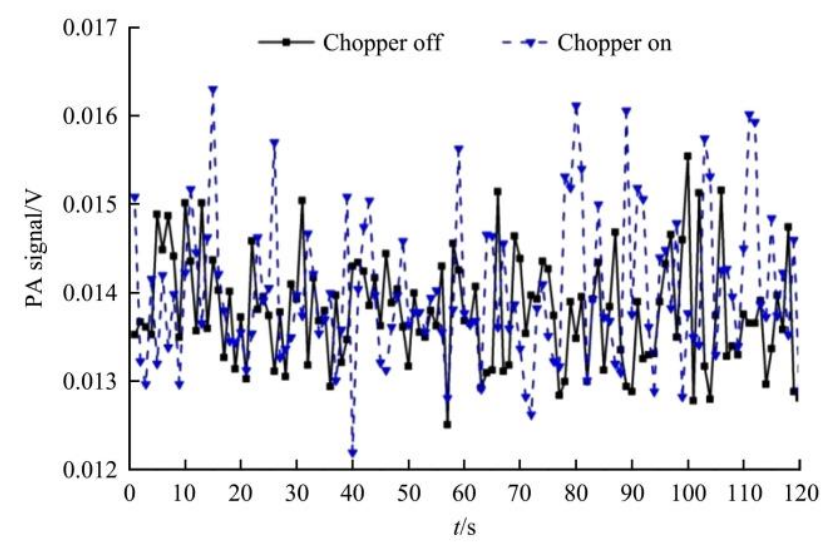

Figure 8 Schematic of noise which is not related to the light source measured experimentally 
In order to analyze whether environmental noise, gas flow noise and electrical noise will affect the stability of the PA signal, relevant experiments are carried out. In the experiment, high purity $\mathrm{N}_{2}$ is filled into the PA cell, the light source and the chopper are turned off, and the data are collected. The experimental time lasts from 10:00 a.m. to the next day at 8:00 a.m. and PAS signals are measured every $20 \mathrm{~min}$. The specific experimental results are shown in Figure 9.

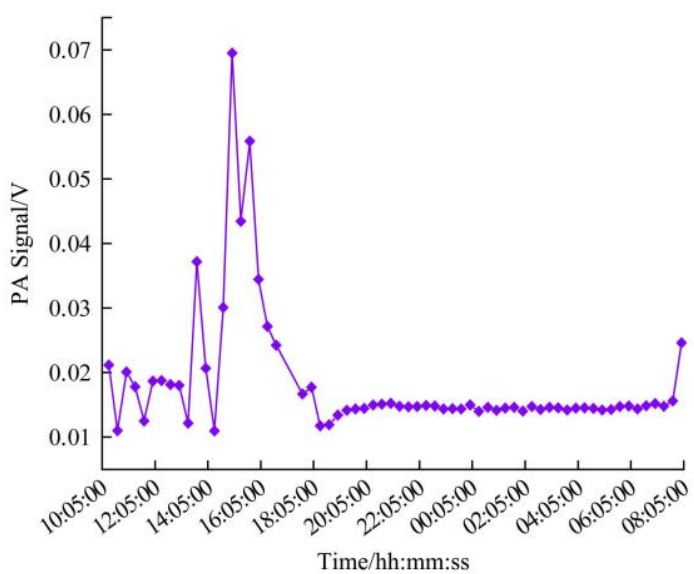

Figure 9 Schematic of variation of environmental noise, gas flow noise and electrical noise with times

From Figure 9, it can be seen that the amplitude of the equivalent PA signal caused by environmental noise, etc. will change with the measurement time. Because the noise of gas flow and electric noise will not change so obviously with time, the environmental noise should change with the time of measurement. From 10:00 a.m. to 2:00 p.m. on the 20th day, the mean and standard deviation of equivalent PA signal caused by environmental noise are $0.02246 \mathrm{~V}$ and $0.01591 \mathrm{~V}$, respectively; from 2:00 p.m. to 6:00 p.m., the amplitudes of equivalent PA signal caused by environmental noise are $0.03311 \mathrm{~V}$ and $0.02026 \mathrm{~V}$; from 6:00 p.m. to 7:40 a.m. on the $21 \mathrm{st}$ day, the mean and standard deviation of equivalent PA signal caused by environmental noise are $0.01473 \mathrm{~V}$ and $3.4832 \times 10^{-4} \mathrm{~V}$, respectively; After 8:00 a.m. on 21 st day, the amplitude of equivalent PA signal caused by environmental noise began to rise again, basically restore to the level of 20th morning. The reason may be that there are sound signals with the same frequency as the modulation frequency of the light source around the laboratory during the day or the vibration propagates through the air to interfere with the measurement of the PA system. This interference is the same frequency as the useful signal and cannot be removed by the phase-locked method. And this kind of interference signal is stronger in the afternoon than in the morning and weakest in the evening. According to the above analysis, this kind of environmental noise with the same frequency as the modulation frequency will affect the stability of PA detection, but because it is a noise signal with the same frequency as the modulation frequency of the light source, it cannot be eliminated by the signal detection technology such as lock-in amplification, and the influence of environmental noise can be minimized by installing sound insulation and muffler.

\section{Conclusions}

Carbon monoxide and sulfur dioxide were used as examples to explain the potential application of PAS technology and analyze the influence mechanism of pressure and noise on the PA signal. Theoretical analysis and experimental studies showed that both pressure and noise had an impact on the stability of the PA signal. Among them, the $\mathrm{CO}$ gas PA signal was proportional to the pressure in the PA cell, and environmental noise with the same frequency of the PA signal was the main noise factor affecting the stability of the PA signal. The analysis showed that the influence of pressure on the PA signal could be corrected by the correction algorithm, and the environmental noise of the same frequency with the PA signal could be eliminated as far as possible by installing sound insulation and silencing devices. In the next step, based on the analysis of the influencing factors, further research will be carried out on the correction algorithm of the pressure and other factors of the PA signal, so as to improve the stability of the PA signal.

\section{Acknowledgements}

The authors acknowledge Professor Daming Dong, National Engineering Research Center for Information Technology in Agriculture, for his helpful suggestions on this work.

\section{[References]}

[1] Li J S, Chen W D, Yu B L. Recent progress on infrared photoacoustic spectroscopy techniques. Applied Spectroscopy Reviews, 2011; 46(6): 440-471.

[2] Bageshwar D V, Pawar A S, Khanvilkar V V, Kadam V J. Photoacoustic Spectroscopy and Its Applications-A Tutorial Review. Eurasian J. Anal. Chem., 2010; 5(2): 187-203.

[3] Karpf A, Rao G N. Enhanced sensitivity for the detection of trace gases using multiple line integrated absorption spectroscopy. Applied Optics, 2009; 48(27): 5061-5066.

[4] Uotila J. Comparison of infrared sources for a differential photoacoustic gas detection system. Infrared Physics \& Technology, 2007; 51(2): 122-130.

[5] Ma Y F. Review of recent advances in QEPAS-based trace gas sensing. Appl. Sci., 2018; 8(10): 1822. doi: 10.3390/app8101822.

[6] Spagnolo V, Patimisco P, Sampaolo A, Giglio M, Tittel F K. Recent advances in quartz enhanced photoacoustic sensing. Appl. Phys. Rev., 2018; 5: 011106. doi: 10.1117/12.2284162.

[7] Gong Z F, Chen K, Chen Y W, Mei L, Yu Q X. Integration of T-type half-open photoacoustic cell and fiber-optic acoustic sensor for trace gas detection. Optics Express, 2019; 27(13): 18222-18231.

[8] Tang J, Zhu L M, Liu F, Fan M. Development of SF6 decomposition components detection device using acoustic technology. High Voltage Engineering, 2011; 37(6): 1313-1320. (in Chinese)

[9] Zhang W, Yu Q X. IR thermal-emitter based photoacoustic spectrometer for gas detection. Spectroscopy and Spectral Analysis, 2007; 27(3): 614-618. (in Chinese)

[10] Gong Y H, Nian S P, Wang C H, Gong R K. Study on detecting gas nitrogen oxides based on research of three-time light path on photoacoustic spectroscopy. Instrument Technique and Sensor, 2014; 10: 85-86, 89. (in Chinese)

[11] Wang J, Du C W, Shen Y Z, Ma F, Zhou J M. Measurement of ammonia in soil headspace by mid-infrared photoacoustic spectroscopy. Soils, 2014; 46(6): 1017-1023. (in Chinese)

[12] Yin X K, Dong L, Zheng H D, Liu X L, Wu H P, Yang Y F, et al. Impact of humidity on quartz-enhanced photoacoustic spectroscopy based $\mathrm{CO}$ detection using a near-IR telecommunication diode laser. Sensors, 2016; 16(2): 162. doi: 10.3390/s16020162.

[13] Yin X K, Dong L, Wu H P, Zheng H D, Ma W G, Zhang L, et al Sub-ppb nitrogen dioxide detection with a large linear dynamic range by use of a differential photoacoustic cell and a $3.5 \mathrm{~W}$ blue multimode diode laser. Sens. Actuators B Chem., 2017; 247: 329-335.

[14] Luo J, Fang Y H, Su Z X, Li D C, Zhao Y D, Wang A J, et al. The research of temperature properties of photoacoustic spectroscopy detection for SF6 decomposition products in gas insulated switchgear. Analytical Methods, 2015; 7(3): 1200-1207.

[15] Ma W G, Yin W B, Huang T, Zhao Y T, Li C Y, Jia S T. Analysis of gas absorption coefficient at various pressures. Spectroscopy and Spectral Analysis, 2004; 24(2): 135-137. (in Chinese) 
[16] Zhao J J, Zhao Z, Du L D, Wu S H. Macro machined photoacoustic non-resonant cell. Key Engineering Materials, 2011; 483: 411-416.

[17] Miklos S, Helga H, Zoltan B, Gabor S. On the pressure dependent sensitivity of a photoacoustic water vapor detector using active laser modulation control. Infrared Physics \& Technology, 2006; 48(3): 192-201

[18] Tavakoli M, Tavakoli A, Taheri M, Saghafifar H. Design, simulation and structural optimization of a longitudinal acoustic resonator for trace gas detection using laser photoacoustic spectroscopy (LPAS). Optics \& Laser Technology, 2010; 42(5): 828-838.

[19] Gondal M A, Dastageer A, Shwehdi M H. Photoacoustic spectroscopy for trace gas analysis and leak detection using different cell geometrics. Talanta, 2004; 62(1): 131-141.

[20] Ulasevich A L, Gorelik A V, Kouzmouk A A, Starovoitov V S. A compact resonant $\Pi$-shaped photoacoustic cell with low window background for gas sensing. Appl. Phys. B, 2014; 117: 549-561.

[21] Liu X Y, Zhou F J, Hu J S, Li H L. Prospect to apply photoacoustic spectroscopy in dissolved gases in oil analysis. Transformer, 2004; 41(7): 30-33. (in Chinese)

[22] Kapp J, Weber C, Schmitt K, Pernau H F, Wöllenstein J. Resonant photoacoustic spectroscopy of $\mathrm{NO}_{2}$ with a UV-LED based sensor. Sensors, 2019; 19(3): 724 . doi: 10.3390/s19030724. 